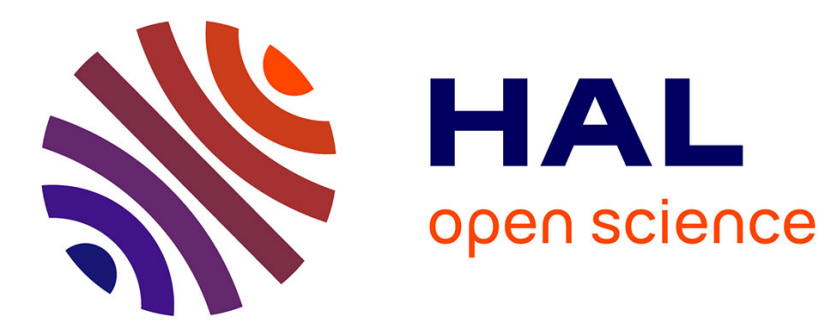

\title{
Bayesian fusion of multispectral and hyperspectral images with unknown sensor spectral response
}

\author{
Qi Wei, Nicolas Dobigeon, Jean-Yves Tourneret
}

\section{To cite this version:}

Qi Wei, Nicolas Dobigeon, Jean-Yves Tourneret. Bayesian fusion of multispectral and hyperspectral images with unknown sensor spectral response. IEEE International Conference on Image Processing (ICIP 2014), Oct 2014, Paris, France. pp. 698-702. hal-01399867

\section{HAL Id: hal-01399867 https://hal.science/hal-01399867}

Submitted on 21 Nov 2016

HAL is a multi-disciplinary open access archive for the deposit and dissemination of scientific research documents, whether they are published or not. The documents may come from teaching and research institutions in France or abroad, or from public or private research centers.
L'archive ouverte pluridisciplinaire HAL, est destinée au dépôt et à la diffusion de documents scientifiques de niveau recherche, publiés ou non, émanant des établissements d'enseignement et de recherche français ou étrangers, des laboratoires publics ou privés. 


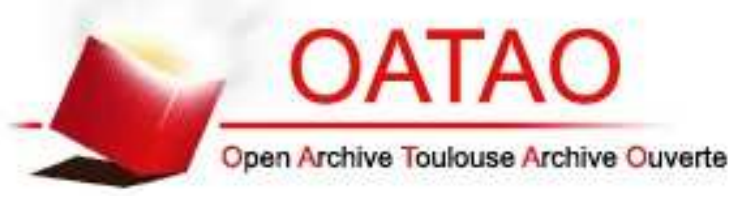

\section{Open Archive TOULOUSE Archive Ouverte (OATAO)}

OATAO is an open access repository that collects the work of Toulouse researchers and makes it freely available over the web where possible.

This is an author-deposited version published in : http://oatao.univ-toulouse.fr/ Eprints ID : 15173

The contribution was presented at ICIP 2014 :

https://icip2014.wp.mines-telecom.fr/

To cite this version : Wei, Qi and Dobigeon, Nicolas and Tourneret, Jean-Yves Bayesian fusion of multispectral and hyperspectral images with unknown sensor spectral response. (2015) In: IEEE International Conference on Image Processing (ICIP 2014), 27 October 2014 - 30 October 2014 (Paris, France).

Any correspondence concerning this service should be sent to the repository administrator: staff-oatao@listes-diff.inp-toulouse.fr 


\title{
BAYESIAN FUSION OF MULTISPECTRAL AND HYPERSPECTRAL IMAGES WITH UNKNOWN SENSOR SPECTRAL RESPONSE
}

\author{
Qi Wei, Nicolas Dobigeon, and Jean-Yves Tourneret \\ University of Toulouse, IRIT/INP-ENSEEIHT, 31071 Toulouse cedex 7, France
}

\begin{abstract}
This paper studies a new Bayesian algorithm for fusing hyperspectral and multispectral images. The observed images are related to the high spatial resolution hyperspectral image to be recovered through physical degradations, e.g., spatial and spectral blurring and/or subsampling defined by the sensor characteristics. In this work, we assume that the spectral response of the multispectral sensor is unknown as it may not be available in practical applications. The resulting fusion problem is formulated within a Bayesian estimation framework, which is very convenient to model the uncertainty regarding the multispectral sensor characteristics and the scene to be estimated. The high spatial resolution hyperspectral image is then inferred from its posterior distribution. More precisely, to compute the Bayesian estimators associated with this posterior, a Markov chain Monte Carlo algorithm is proposed to generate samples asymptotically distributed according to the distribution of interest. Simulation results demonstrate the efficiency of the proposed fusion method when compared with several state-of-the-art fusion techniques.
\end{abstract}

Index Terms - Fusion, multispectral and hyperspectral images, spectral response, Bayesian estimation, Hamiltonian Monte Carlo.

\section{INTRODUCTION}

Multi-resolution image fusion, also known as super-resolution, has been a very active research topic during recent years [1]. A prototypal fusion task for remote sensing images is the pansharpening, which generally consists of fusing a high spatial resolution panchromatic (PAN) image and a low spatial resolution multispectral (MS) image. More recently, hyperspectral (HS) imaging, which consists of acquiring a same scene in several hundreds of contiguous spectral bands, has opened a new range of relevant applications, such as target detection [2] and spectral unmixing [3]. Naturally, to take advantage of the newest benefits offered by HS images, the problem of fusing HS and PAN images has been explored [4]. Capitalizing on decades of experience in MS pansharpening, several HS pansharpening approaches merely adapt existing algorithms for PAN and MS fusion [5]. Other methods are specifically designed to the HS pansharpening problem such as [6]. Conversely, the fusion of MS and HS images has been considered in fewer research works and is still a challenging problem because of the high dimensionality of the data to be processed. The fusion of MS and HS differs from traditional MS or HS pansharpening since both spatial and spectral information is contained in multi-band images. Therefore, a lot of pansharpening methods, such as component substitution [7] and relative spectral contribution [8] are inapplicable or inefficient for the HS/MS fusion

Part of this work has been supported by the Chinese Scholarship Council (CSC, 201206020007), the Hypanema ANR Project $n^{\circ}$ ANR-12-BS03-003 and ANR-11-LABX-0040-CIMI in particular during the program ANR-11IDEX-0002-02 within the thematic trimester on image processing. problem. Since the fusion problem is ill-posed, Bayesian inference offers a convenient way to regularize the problem by defining an appropriate prior distribution for the scene of interest. Following this strategy, Hardie et al. proposed a Bayesian estimator for fusing the co-registered high spatial-resolution MS and high spectralresolution HS images [9]. The estimator of [9] was implemented by Zhang et al. in the wavelet domain to improve denoising performance [10]. More recently, a hierarchical Bayesian model was proposed in $[11,12]$ to solve the fusion problem. The Bayesian estimators associated with this model were computed from samples generated from the target posterior distribution using Markov chain Monte Carlo methods. However, the spectral and spatial sensor responses were assumed to be fully known in [11] and were exploited to properly design the spatial and spectral degradations affecting the image to be recovered (see [13] for more details about these degradations). In practice, the spectral relation between HS and MS images is not always available as the corresponding spectral responses highly rely on the sensors.

In this work, we propose to estimate the spectral response of the MS sensor jointly with the unknown image to be recovered, generalizing the approach of [11]. Exploiting the intrinsic dimension of the data to be recovered, the MS characteristics are expressed in a lower-dimensional subspace, significantly reducing the difficulties inherent to the resulting blind deconvolution problem. Based on the posterior distribution of the unknown parameters, we propose to compute the MMSE estimators of the unknown scene and a so-called pseudo-spectral response by using samples generated by a hybrid Gibbs sampler. This sampler includes a Hamiltonian Monte Carlo (HMC) step. The HMC algorithm differs from the standard Metropolis-within-Gibbs algorithm by exploiting Hamiltonian evolution dynamics to propose states in a high-dimensional space with higher acceptance ratio, reducing the correlation between successive samples and thus speeding up the sampler convergence.

The paper is organized as follows. Section 2 formulates the fusion problem in a Bayesian framework. In Section 3, we propose a new hierarchical Bayesian model defined by the joint posterior distribution of the unknown image, its hyperparameters, the pseudospectral response and the noise variances. Section 4 studies a hybrid Gibbs sampler based on an HMC method to sample the target joint posterior distribution of interest. Simulation results are presented in Section 5 whereas conclusions are reported in Section 6.

\section{PROBLEM FORMULATION}

In this paper, we consider the problem of fusing HS and MS images. As mentioned before, the HS image $\mathbf{Y}_{\mathrm{H}}$ is supposed to be a blurred, down-sampled and noisy version of the target image $\mathbf{X}$ whereas the MS image $\mathbf{Y}_{\mathrm{M}}$ is a spectrally degraded and noisy version of $\mathbf{X}$. As a consequence, the observation models associated with the HS and MS images can be written as $[9,14,15]$

$$
\begin{aligned}
& \mathbf{Y}_{\mathrm{H}}=\mathbf{X B S}+\mathbf{N}_{\mathrm{H}} \\
& \mathbf{Y}_{\mathrm{M}}=\mathbf{R X}+\mathbf{N}_{\mathrm{M}}
\end{aligned}
$$


where $\mathbf{X}=\left[\boldsymbol{x}_{1}, \cdots, \boldsymbol{x}_{n}\right] \in \mathbb{R}^{m_{\lambda} \times n}$ is the unknown full resolution HS image composed of $m_{\lambda}$ bands and $n$ pixels, $\mathbf{Y}_{\mathrm{H}} \in \mathbb{R}^{m_{\lambda} \times m}$ is the HS image composed of $m_{\lambda}$ bands and $m$ pixels and $\mathbf{Y}_{\mathrm{M}} \in$ $\mathbb{R}^{n_{\lambda} \times n}$ is the MS image composed of $n_{\lambda}$ bands and $n$ pixels. In (2), $\mathbf{B} \in \mathbb{R}^{n \times n}$ is a cyclic convolution operator acting on the bands that models the point spread function of the HS sensor and $\mathbf{S} \in \mathbb{R}^{n \times m}$ is a downsampling matrix (with downsampling factor denoted as $d$ ). Conversely, $\mathbf{R} \in \mathbb{R}^{n_{\lambda} \times m_{\lambda}}$ models the spectral response of the MS sensor, which is assumed to be unknown. The noise matrices $\mathbf{N}_{\mathrm{H}} \in$ $\mathbb{R}^{m_{\lambda} \times m}$ and $\mathbf{N}_{\mathrm{M}} \in \mathbb{R}^{n_{\lambda} \times n}$ are assumed to be distributed according to the following matrix Gaussian distributions [16]

$$
\begin{aligned}
& \mathbf{N}_{\mathrm{H}} \sim \mathcal{M} \mathcal{N}_{m_{\lambda}, m}\left(\mathbf{0}_{m_{\lambda}, m}, s_{h}^{2} \mathbf{I}_{m_{\lambda}}, \mathbf{I}_{m}\right) \\
& \mathbf{N}_{\mathrm{M}} \sim \mathcal{M} \mathcal{N}_{n_{\lambda}, n}\left(\mathbf{0}_{n_{\lambda}, n}, s_{m}^{2} \mathbf{I}_{n_{\lambda}}, \mathbf{I}_{n}\right)
\end{aligned}
$$

where $\mathbf{0}_{a, b}$ is the $a \times b$ matrix of zeros and $\mathbf{I} .{ }_{\lambda}$ is the $\cdot_{\lambda} \times{ }_{\lambda}$ identity matrix. Note that the white Gaussian noise assumption is quite popular in image processing $[17,18]$ as it facilitates the formulation of the likelihood. However, the proposed work could be extended to handle colored noise, in particular to cope with spectrally correlated noise. The problem addressed in this work consists of estimating the high-spatial resolution HS image $\mathbf{X}$ from the two available images $\mathbf{Y}_{\mathrm{H}}$ and $\mathbf{Y}_{\mathrm{M}}$, using the observation model (2) when the spectral response matrix $\mathbf{R}$ is unknown. The proposed estimation scheme relies on a hierarchical Bayesian model introduced in Section 3.

\section{HIERARCHICAL BAYESIAN MODEL}

\subsection{Reformulation in a lower-dimensional subspace}

Because the HS bands are spectrally correlated, the HS vector $\boldsymbol{x}_{i}$ usually lives in a space whose dimension is much smaller than $m_{\lambda}$ [19]. This property has been extensively exploited when analyzing HS data, in particular to perform spectral unmixing [3]. More precisely, the HS image can be rewritten as $\mathbf{X}=\mathbf{V U}$ where $\mathbf{V} \in$ $\mathbb{R}^{m_{\lambda} \times \tilde{m}_{\lambda}}$ has normalized orthogonal columns and $\mathbf{U} \in \mathbb{R}^{\tilde{m}_{\lambda} \times n}$ is the projection of $\mathbf{X}$ onto the subspace spanned by the columns of $\mathbf{V}$. Incorporating this decomposition of the HS image $\mathbf{X}$ into the observation model (2) leads to

$$
\begin{aligned}
& \mathbf{Y}_{\mathrm{H}}=\mathbf{V} \mathbf{U B S}+\mathbf{N}_{\mathrm{H}} \\
& \mathbf{Y}_{\mathrm{M}}=\widetilde{\mathbf{R}} \mathbf{U}+\mathbf{N}_{\mathrm{M}}
\end{aligned}
$$

where $\widetilde{\mathbf{R}} \triangleq \mathbf{R V}$ is the so-called pseudo-spectral response of the MS sensor. Since $\mathbf{V}$ is a full-column rank matrix, the rows of $\mathbf{V}$ span the space $\mathbb{R}^{\widetilde{m}_{\lambda} \times 1}$, which implies that the rows of $\widetilde{\mathbf{R}}=\mathbf{R V}$ also live in $\mathbb{R}^{\tilde{m}_{\lambda} \times 1}$. Thus, without loss of generality, the estimation of the full spectral response $\mathbf{R}$ can be substituted by the estimation of the pseudo-response $\widetilde{\mathbf{R}}$, which significantly decreases the computational complexity of the fusion algorithm since $\widetilde{m}_{\lambda} \ll m_{\lambda}$. In this work, we assume that the signal subspace denoted as $\operatorname{span}\{\mathbf{V}\}$ has been previously identified, e.g., obtained from available a priori knowledge regarding the scene of interest, or after conducting a principal component analysis (PCA) of the HS data. Then, the considered fusion problem is solved in this lower-dimensional subspace, by estimating the projected image $\mathbf{U}$ and the pseudo-spectral response $\widetilde{\mathbf{R}}$.

\subsection{Likelihood and prior distributions}

Using the statistical properties of the matrices $\mathbf{N}_{H}$ and $\mathbf{N}_{M}$, the distributions of $\mathbf{Y}_{\mathrm{H}}$ and $\mathbf{Y}_{\mathrm{M}}$ are matrix Gaussian distributions, i.e.,

$$
\begin{aligned}
& \mathbf{Y}_{\mathrm{H}} \sim \mathcal{M N _ { m _ { \lambda } , m }}\left(\mathbf{V U B S}, s_{h}^{2} \mathbf{I}_{m_{\lambda}}, \mathbf{I}_{m}\right), \\
& \mathbf{Y}_{\mathrm{M}} \sim \mathcal{M} \mathcal{N}_{n_{\lambda}, n}\left(\mathbf{R V U}, s_{m}^{2} \mathbf{I}_{n_{\lambda}}, \mathbf{I}_{n}\right) .
\end{aligned}
$$

The unknown parameters to be estimated are the projected scene $\mathbf{U}$, the pseudo-spectral response $\widetilde{\mathbf{R}}$ and the vector of noise variances $s^{2}=\left\{s_{h}^{2}, s_{m}^{2}\right\}$. The appropriate prior distributions assigned to these parameters are presented below.

Scene prior: Gaussian prior distributions are assigned to the projected vectors $\boldsymbol{u}_{i}(i=1, \cdots, n)$ that are assumed to be a priori independent, i.e.,

$$
\boldsymbol{u}_{i} \mid \boldsymbol{\mu}_{\boldsymbol{u}_{i}}, \boldsymbol{\Sigma}_{\boldsymbol{u}_{i}} \sim \mathcal{N}\left(\boldsymbol{\mu}_{\boldsymbol{u}_{i}}, \boldsymbol{\Sigma}_{\boldsymbol{u}_{i}}\right)
$$

where $\boldsymbol{\mu}_{\boldsymbol{u}_{i}}$ are fixed using the interpolated HS image in the subspace of interest following the strategy in [9] and $\boldsymbol{\Sigma}_{\boldsymbol{u}_{i}}$ are unknown hyperparameters. To reduce the number of parameters to be estimated, $\boldsymbol{\Sigma}_{\boldsymbol{u}_{i}}$ are assumed to be identical, i.e., $\boldsymbol{\Sigma}_{\boldsymbol{u}_{1}}=\cdots=\boldsymbol{\Sigma}_{\boldsymbol{u}_{n}}=\boldsymbol{\Sigma}_{\boldsymbol{u}}$.

The Gaussian prior has been used successfully in many image processing applications including image denoising [20] and image restoration [21]. Moreover, it has the advantage of being a conjugate distribution relative to the likelihood function, leading to simple computations of the Bayesian estimators derived from the posterior distribution of interest.

Pseudo-spectral response prior: A matrix Gaussian prior is chosen for $\widetilde{\mathbf{R}}$, i.e., $p\left(\widetilde{\mathbf{R}} \mid \overline{\mathbf{R}}, \sigma_{\mathbf{R}}^{2}\right)=\mathcal{M N}_{n_{\lambda}, \widetilde{m}_{\lambda}}\left(\overline{\mathbf{R}}, \sigma_{\mathbf{R}}^{2} \mathbf{I}_{n_{\lambda}}, \mathbf{I}_{\widetilde{m}_{\lambda}}\right)$. In absence of additional knowledge, the mean response $\overline{\mathbf{R}}$ is set to the zero matrix and $\sigma_{\mathbf{R}}^{2}$ is set to a large value to ensure a non-informative prior for $\widetilde{\mathbf{R}}$.

Noise variance priors: A non-informative Jeffreys' prior is assigned to the noise variances $s_{h}^{2}$ and $s_{m}^{2}$, i.e., $f\left(s_{h}^{2}\right) \propto \frac{1}{s_{h}^{2}} \mathbf{1}_{\mathbb{R}^{+}}\left(s_{h}^{2}\right)$ and $f\left(s_{m}^{2}\right) \propto \frac{1}{s_{m}^{2}} \mathbf{1}_{\mathbb{R}^{+}}\left(s_{m}^{2}\right)$, where $\mathbf{1}_{\mathbb{R}^{+}}(\cdot)$ is the indicator function defined on $\mathbb{R}^{+}$(see [22] for motivations).

\subsection{Hyperparameter priors}

The hyperparameter vector associated with the parameter priors defined above is $\boldsymbol{\Phi}=\left\{\boldsymbol{\Sigma}_{\boldsymbol{u}}\right\}$. The quality of the fusion algorithm investigated in this paper clearly depends on the value of this hyperparameter. Instead of fixing the hyperparameter a priori, we propose to estimate it from the data by defining a hierarchical Bayesian model. This approach requires to define prior for the this hyperparameter (usually referred to as hyperprior) which is detailed below.

Hyperparameter $\Sigma_{u}$ : Assigning a conjugate inverse-Wishart (IW) distribution to the covariance matrix $\boldsymbol{\Sigma}_{\boldsymbol{u}}$ has provided interesting results in the signal/image processing literature [23]. Following these works, an IW distribution $\boldsymbol{\Sigma}_{\boldsymbol{u}} \sim \mathcal{I} \mathcal{W}(\boldsymbol{\Psi}, \eta)$ has been chosen, where the parameters $(\Psi, \eta)^{T}$ are fixed to provide a non-informative prior for $\boldsymbol{\Sigma}_{u}$.

\subsection{Posterior distribution}

The unknown parameter vector $\boldsymbol{\theta}$ associated with the proposed hierarchical Bayesian fusion model is composed of the projected scene $\mathbf{U}$, the pseudo-spectral response $\widetilde{\mathbf{R}}$ and the noise variances $s^{2}$, i.e., $\boldsymbol{\theta}=\left\{\mathbf{U}, \widetilde{\mathbf{R}}, s^{2}\right\}$. Defining $\mathcal{Y}=\left\{\mathbf{Y}_{\mathrm{H}}, \mathbf{Y}_{\mathrm{M}}\right\}$ the set of the observed images, the joint posterior distribution of the unknown parameters and hyperparameters can be computed using the following hierarchical structure

$$
f(\boldsymbol{\theta}, \boldsymbol{\Phi} \mid \mathcal{Y}) \propto f(\mathcal{Y} \mid \boldsymbol{\theta}) f(\boldsymbol{\theta} \mid \boldsymbol{\Phi}) f(\boldsymbol{\Phi})
$$


where the parameter and hyperparameter priors are given by

$$
\begin{array}{ll}
f(\boldsymbol{\theta} \mid \boldsymbol{\Phi}) & =f\left(\mathbf{U} \mid \boldsymbol{\Sigma}_{\boldsymbol{u}}\right) f(\widetilde{\mathbf{R}}) f\left(s_{h}^{2}\right) f\left(s_{m}^{2}\right) \\
f(\boldsymbol{\Phi}) & =f\left(\boldsymbol{\Sigma}_{\boldsymbol{u}}\right) .
\end{array}
$$

Computing the posterior distribution of the projected scene $f(\mathbf{U} \mid \mathcal{Y})$ requires to marginalize out the parameters $\Phi, \widetilde{\mathbf{R}}$ and $s^{2}$ from the joint posterior. As this marginalization is clearly not easy to perform, computing the MMSE and MAP estimators of the projected scene $\mathbf{U}$ analytically from the posterior (6) is difficult. Instead, this paper proposes to generate a collection of $N_{\mathrm{MC}}$ samples $\left\{(\boldsymbol{\theta}, \boldsymbol{\Phi})^{(1)}, \ldots,(\boldsymbol{\theta}, \boldsymbol{\Phi})^{\left(N_{\mathrm{MC}}\right)}\right\}$ that are asymptotically distributed according to the posterior of interest (6). The Bayesian estimators of the parameters of interest can then be computed using these generated samples. For instance, the MMSE estimator of $\mathbf{U}$ can be approximated by an empirical average of the generated samples $\hat{\mathbf{U}}_{\mathrm{MMSE}} \approx \frac{1}{N_{\mathrm{MC}}-N_{\mathrm{bi}}} \sum_{t=N_{\mathrm{bi}}+1}^{N_{\mathrm{MC}}} \mathbf{U}^{(t)}$, where $N_{\mathrm{bi}}$ is the number of burn-in iterations required to reach the sampler convergence. The highly-resolved HS image can finally be computed as $\hat{\mathbf{X}}_{\mathrm{MMSE}}=\mathbf{V} \hat{\mathbf{U}}_{\mathrm{MMSE}}$. In order to sample according to the joint posterior $f(\boldsymbol{\theta}, \boldsymbol{\Phi} \mid \mathcal{Y})$, we propose to design a Metropolis-withinGibbs sampler whose main steps are described in the next section.

\section{HYBRID GIBBS SAMPLER}

The Gibbs sampler has received much attention in the statistical community to solve Bayesian estimation problems [24]. The main idea of this MCMC method is to sample according to the conditional distributions of the target distribution (see [24] for more details). The sampler is defined by a 4 -step procedure detailed below.

\subsection{Sampling the covariance matrix of the image $\Sigma_{u}$}

Standard computations yield the following inverse-Wishart distribution as conditional distribution for the covariance matrix $\boldsymbol{\Sigma}_{u}$

$$
\begin{aligned}
& \boldsymbol{\Sigma}_{\boldsymbol{u}} \mid \mathbf{U}, \widetilde{\mathbf{R}}, \boldsymbol{s}^{2}, \mathcal{Y} \sim \\
& \quad \mathcal{I} \mathcal{W}\left(\boldsymbol{\Psi}+\sum_{i=1}^{n}\left(\boldsymbol{u}_{i}-\boldsymbol{\mu}_{\boldsymbol{u}_{i}}\right)^{T}\left(\boldsymbol{u}_{i}-\boldsymbol{\mu}_{\boldsymbol{u}_{i}}\right), n+\eta\right)
\end{aligned}
$$

which is easy to sample.

\subsection{Sampling the pseudo-spectral response matrix $\widetilde{\mathbf{R}}$}

The conditional PDF of $\widetilde{\mathbf{R}}$ can be computed using the likelihood (4) and the prior defined in Section 3.2. We obtain $\widetilde{\mathbf{R}} \mid \boldsymbol{\Sigma}_{\boldsymbol{u}}, \mathbf{U}, \boldsymbol{s}^{2}, \mathcal{Y} \sim$ $\mathcal{M N}_{n_{\lambda}, \widetilde{m}_{\lambda}}\left(\boldsymbol{\mu}_{\widetilde{\mathbf{R}}}, \mathbf{I}_{n_{\lambda}}, \boldsymbol{\Sigma}_{\widetilde{\mathbf{R}}}\right)$ with

$$
\begin{aligned}
& \boldsymbol{\mu}_{\widetilde{\mathbf{R}}}=\left(\frac{1}{s_{m}^{2}} \mathbf{Y}_{\mathrm{M}} \mathbf{U}^{T}+\frac{1}{\sigma_{\mathbf{R}}^{2}} \overline{\mathbf{R}}\right) \boldsymbol{\Sigma}_{\widetilde{\mathbf{R}}} \\
& \boldsymbol{\Sigma}_{\widetilde{\mathbf{R}}}=\left(\frac{1}{s_{m}^{2}} \mathbf{U} \mathbf{U}^{T}+\frac{1}{\sigma_{\mathbf{R}}^{2}} \mathbf{I}_{\widetilde{m}_{\lambda}}\right)^{-1}
\end{aligned}
$$

which can be sampled easily. Note in particular that the matrix $\boldsymbol{\Sigma}_{\widetilde{\mathbf{R}}} \in \mathbb{R}^{\widetilde{m}_{\lambda} \times \tilde{m}_{\lambda}}$ can be computed easily since it has a small size $\left(\widetilde{m}_{\lambda}\right.$ is generally smaller than 10$)$.

\subsection{Sampling the projected image $U$}

Choosing the prior distribution for the projected image $\mathbf{U}$ defined in Section 3.2 leads to the conditional log-posterior distribution

$$
\begin{aligned}
& -\log f\left(\mathbf{U} \mid \boldsymbol{\Sigma}_{\boldsymbol{u}}, \widetilde{\mathbf{R}}, \boldsymbol{s}^{2}, \mathcal{Y}\right)=\frac{1}{2 s_{h}^{2}}\left\|\mathbf{Y}_{\mathrm{H}}-\mathbf{V U B S}\right\|_{F}^{2}+ \\
& \frac{1}{2 s_{m}^{2}}\left\|\mathbf{Y}_{\mathrm{M}}-\widetilde{\mathbf{R}} \mathbf{U}\right\|_{F}^{2}+\frac{1}{2} \sum_{i=1}^{n}\left(\boldsymbol{u}_{i}-\boldsymbol{\mu}_{\boldsymbol{u}_{i}}\right)^{T} \boldsymbol{\Sigma}_{\boldsymbol{u}}^{-1}\left(\boldsymbol{u}_{i}-\boldsymbol{\mu}_{\boldsymbol{u}_{i}}\right)+\mathrm{C}
\end{aligned}
$$

where $\|\cdot\|_{F}$ is the Frobenius norm and $\mathrm{C}$ does not depend on $\mathbf{U}$. Note that the vector obtained by vectorizing $\mathbf{U}$ has a Gaussian distribution. However, $f\left(\mathbf{U} \mid \boldsymbol{\Sigma}_{u}, \widetilde{\mathbf{R}}, \boldsymbol{s}^{2}, \mathcal{Y}\right)$ is not the PDF of a matrix normal distribution. Therefore, sampling $\mathbf{U}$ directly from its conditional distribution would be computationally intensive, since it would require the inversion of large matrices. In this paper, we propose to use an HMC method to generate matrices distributed according to the conditional distribution of $\mathbf{U}$. More details about the proposed HMC method are available in [11] and are omitted here for space limitations.

\subsection{Sampling the noise variance vector $s^{2}$}

The conditional distributions of the noise variances $s_{h}^{2}$ and $s_{m}^{2}$ are the following inverse-gamma (IG) distributions

$$
\begin{gathered}
s_{h}^{2} \mid \boldsymbol{\Sigma}_{\boldsymbol{u}}, \mathbf{U}, \widetilde{\mathbf{R}}, \mathcal{Y} \sim \mathcal{I} \mathcal{G}\left(\frac{m_{\lambda} m}{2}, \frac{\left\|\mathbf{Y}_{\mathrm{H}}-\mathrm{VUBS}\right\|_{2}^{2}}{2}\right) \\
s_{m}^{2} \mid \boldsymbol{\Sigma}_{\boldsymbol{u}}, \mathbf{U}, \widetilde{\mathbf{R}}, \mathcal{Y} \sim \mathcal{I} \mathcal{G}\left(\frac{n_{\lambda} n}{2}, \frac{\left\|\mathbf{Y}_{\mathrm{M}}-\widetilde{\mathbf{R}}\right\|_{2}^{2}}{2}\right)
\end{gathered}
$$

that are easy to sample.

\section{SIMULATION RESULTS}

This section presents numerical results obtained with the proposed Bayesian fusion algorithm. The reference image, considered here as the high spatial and high spectral resolution image to be recovered, is an HS image acquired over Moffett field, CA, in 1994 by the JPL/NASA airborne visible/infrared imaging spectrometer (AVIRIS) [25]. This image is of size $128 \times 64$ and was initially composed of 224 bands that have been reduced to 177 bands after removing the water vapor absorption bands. A composite color image of the scene of interest is shown in the bottom right of Fig. 1.

\subsection{Simulation scenario}

We propose to reconstruct the reference HS image $\mathbf{X}$ from two HS and MS images $\mathbf{Y}_{\mathrm{H}}$ and $\mathbf{Y}_{\mathrm{M}}$. First, a high-spectral and low-spatial resolution image $\mathbf{Y}_{\mathrm{H}}$ has been generated by applying a $5 \times 5$ averaging filter and by down-sampling every 4 pixels in both vertical and horizontal direction for each band of the reference image. Second, a 7-band MS image $\mathbf{Y}_{\mathrm{M}}$ has been obtained by filtering $\mathbf{X}$ with the LANDSAT reflectance spectral responses [26].

The HS and MS images have been both contaminated by zeromean additive Gaussian noises with signal to noise ratios $\mathrm{SNR}_{\mathrm{H}}=$ $10 \log \left(\frac{\|\mathbf{X B S}\|_{F}^{2}}{\left\|\mathbf{N}_{\mathrm{H}}\right\|_{F}^{2}}\right)=30 \mathrm{~dB}$ and $\mathrm{SNR}_{\mathrm{M}}=10 \log \left(\frac{\|\mathbf{R X}\|_{F}^{2}}{\left\|\mathbf{N}_{\mathrm{M}}\right\|_{F}^{2}}\right)=$ 30dB. The observed HS and MS images are shown in the top left and right of Fig. 1. Note that the HS image has been interpolated for better visualization and that the MS image has been displayed using an arbitrary color composition. In order to learn the projection matrix $\mathbf{V}$, we have computed the $\widetilde{m}_{\lambda}=10$ most discriminant vectors (associated with the 10 largest eigenvalues of the sample covariance matrix) of the HS image. These 10 vectors correspond to $99.89 \%$ of the information contained in the HS image.

\subsection{Hyperparameter Selection}

As presented in Section 3, some prior parameters are fixed to generate a non-informative prior as detailed below:

- $\sigma_{\mathbf{R}}^{2}$ is equal to $10^{3}$ to provide a non-informative prior for the spectral response.

- $\Psi$ is fixed to the identical matrix and $\eta$ is fixed to be $\widetilde{m}_{\lambda}+3$ to ensure a non-informative prior for $\boldsymbol{\Sigma}_{\boldsymbol{u}}$. 

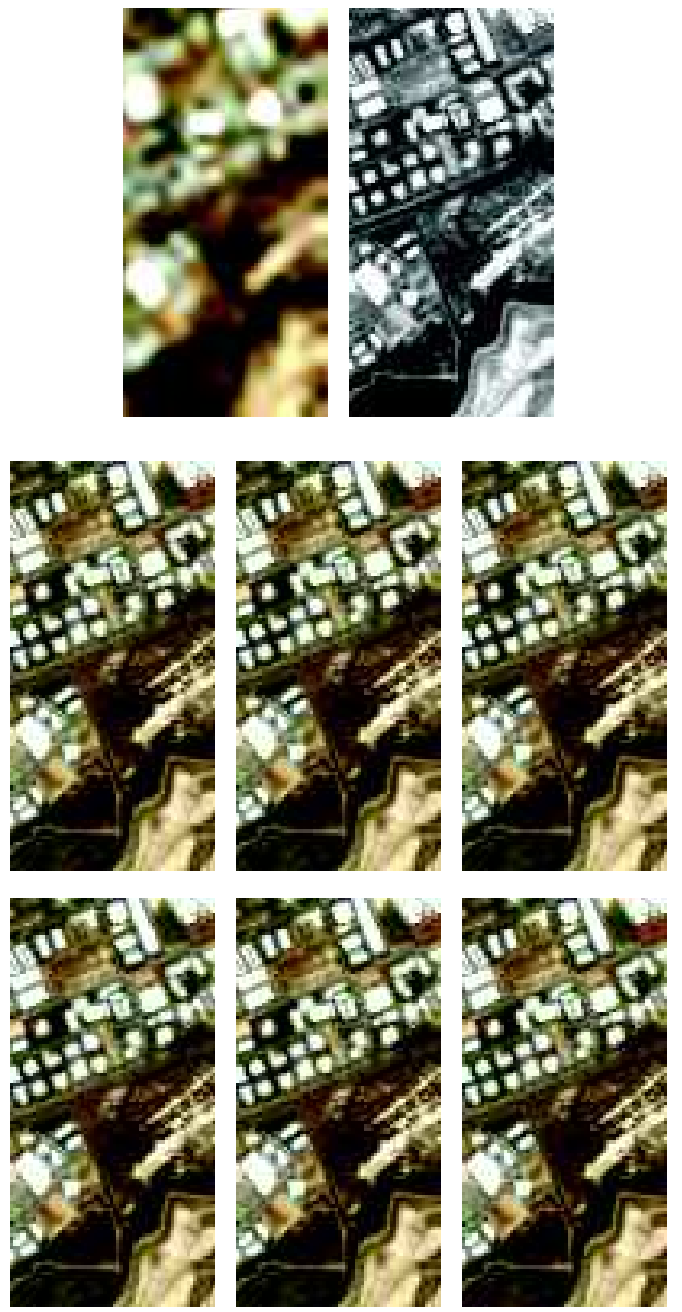

Fig. 1. Fusion results. (Top left) HS image. (Top right) MS image. (Row 2 left) MAP estimator [9]. (Row 2 middle) Wavelet MAP estimator [10]. (Row 2 right) MMSE estimator with known $\mathbf{R}$. (Bottom left) MMSE estimator with $\mathbf{R}+$ noise. (Bottom middle) Proposed method. (Bottom right) Reference image.

\subsection{Fusion performance}

To evaluate the quality of the proposed fusion strategy, three image quality measures have been investigated. Referring to [10], we propose to use the reconstruction SNR (RSNR), the averaged spectral angle mapper (SAM) and the universal image quality index (UIQI) as quantitative measures. The RSNR is related to the Euclidean distance between the actual and fused images $\operatorname{RSNR}(\mathbf{X}, \hat{\mathbf{X}})=$ $10 \log _{10}\left(\frac{\|\mathbf{X}\|_{2}^{2}}{\|\mathbf{X}-\hat{\mathbf{X}}\|_{F}^{2}}\right)$. The larger RSNR, the better the fusion. The definition of SAM and UIQI can be found in [10]. The smaller SAM, the better the fusion and the larger UIQI, the better the fusion.

The experiments compare the proposed hierarchical Bayesian method with three state-of-the-art fusion algorithms for MS and HS images [9-11]. Note that the Bayesian method of [11] mainly differs from the proposed strategy in that the spectral response $\mathbf{R}$ was perfectly known in [11] while it is estimated in this work. Consequently, the algorithm of [11] can be considered as an oracle method
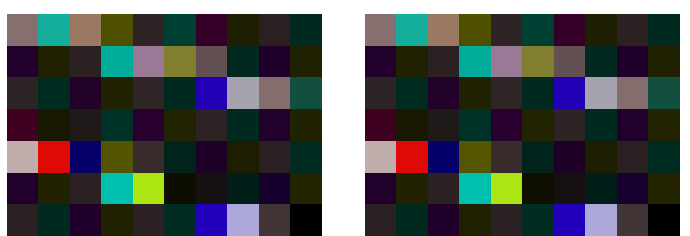

Fig. 2. True pseudo-spectral response $\widetilde{\mathbf{R}}$ (left) and its estimation (right).

Table 1. Performance of the fusion methods: RSNR (in dB), UIQI and SAM (in degree).

\begin{tabular}{c|c|c|c}
\hline Methods & RSNR & UIQI & SAM \\
\hline Hardie [9] & 23.32 & 0.9913 & 5.06 \\
Zhang [10] & 25.42 & 0.9955 & 4.03 \\
MCMC with exact R [11] & 26.57 & 0.9965 & 3.47 \\
MCMC with noisy R & 25.93 & 0.9957 & 3.59 \\
MCMC with unknown R & 26.56 & 0.9965 & 3.46 \\
\hline
\end{tabular}

that can be employed with an exact knowledge of $\mathbf{R}$. Results obtained with the different algorithms are depicted in Fig. 1. The proposed algorithm performs competitively with the other methods for MS and HS fusion. Quantitative results reported in Table 1 in terms of RSNR, SAM and UIQI show that the proposed method provides better results than the methods of [9], [10] and similar performance when compared to the oracle method [11]. Table 1 also shows that the performance of the oracle method [11] degrades heavily when using a spectral response with some uncertainty (obtained by adding noise with variance $\sigma_{\mathbf{R}}^{2}$ defined by $\left.10 \log _{10}\left(\|\mathbf{R}\|_{F}^{2} / \sigma_{\mathbf{R}}^{2}\right)=10 \mathrm{~dB}\right)$. An advantage of the proposed method is that it allows the pseudospectral response of the MS sensor $\widetilde{\mathbf{R}}$ to be estimated. Fig. 2 shows that the resulting estimated $\widetilde{\mathbf{R}}$ is in good agreement with the true pseudo-spectral response (obtained by multiplying the spectral response of the LANDSAT satellite [26] by the matrix $\mathbf{V}$ defined in Section 5.1). Note that the original spectral response $\mathbf{R}$ is not easy to be estimated from $\widetilde{\mathbf{R}}$ since the matrix $\mathbf{V}$ is not invertible.

\section{CONCLUSION}

This paper proposed a new hierarchical Bayesian model for the fusion of multispectral and hyperspectral images when the spectral response of the multispectral sensor is unknown. The image to be recovered was assumed to be degraded by physical transformations included within a forward model. We introduced an appropriate prior distribution for the high spatial and high spectral resolution image to be recovered defined in a lower-dimensional subspace. The resulting posterior distribution was sampled using a hybrid Gibbs sampler. The particularity of this sampler is to involve a Hamiltonian Monte Carlo step for sampling the unknown image, which is projected onto a low dimensional subspace defined by the main vectors of a principal component analysis of the hyperspectral image. Numerical experiments showed that the proposed method compares favorably with other state-of-the-art methods, with the advantage of jointly estimating the spectral response of the multispectral sensor. Future work will consist of comparing the proposed method with other non-Bayesian methods, such as [27-30]. 


\section{REFERENCES}

[1] I. Amro, J. Mateos, M. Vega, R. Molina, and A. K. Katsaggelos, "A survey of classical methods and new trends in pansharpening of multispectral images," EURASIP J. Adv. Signal Process., vol. 2011, no. 1, pp. 1-22, 2011.

[2] D. Manolakis and G. Shaw, "Detection algorithms for hyperspectral imaging applications," IEEE Signal Process. Mag., vol. 19, no. 1, pp. 29-43, jan 2002.

[3] J. M. Bioucas-Dias, A. Plaza, N. Dobigeon, M. Parente, Q. Du, P. Gader, and J. Chanussot, "Hyperspectral unmixing overview: Geometrical, statistical, and sparse regression-based approaches," IEEE J. Sel. Topics Appl. Earth Observations and Remote Sens., vol. 5, no. 2, pp. 354-379, 2012.

[4] M. Cetin and N. Musaoglu, "Merging hyperspectral and panchromatic image data: qualitative and quantitative analysis," Int. J. Remote Sens., vol. 30, no. 7, pp. 1779-1804, 2009.

[5] C. Chisense, J. Engels, M. Hahn, and E. Gülch, "Pansharpening of hyperspectral images in urban areas," in Proc. XXII Congr. of the Int. Society for Photogrammetry, Remote Sens., Melbourne, Australia, 2012.

[6] G. Chen, S.-E. Qian, J.-P. Ardouin, and W. Xie, "Superresolution of hyperspectral imagery using complex ridgelet transform," Int. J. Wavelets, Multiresolution Inf. Process., vol. 10, no. 03, 2012.

[7] V. Shettigara, "A generalized component substitution technique for spatial enhancement of multispectral images using a higher resolution data set," Photogramm. Eng. Remote Sens., vol. 58, no. 5, pp. 561-567, 1992.

[8] J. Zhou, D. Civco, and J. Silander, "A wavelet transform method to merge Landsat TM and SPOT panchromatic data," Int. J. Remote Sens., vol. 19, no. 4, pp. 743-757, 1998.

[9] R. C. Hardie, M. T. Eismann, and G. L. Wilson, "MAP estimation for hyperspectral image resolution enhancement using an auxiliary sensor," IEEE Trans. Image Process., vol. 13, no. 9, pp. 1174-1184, Sept. 2004.

[10] Y. Zhang, S. De Backer, and P. Scheunders, "Noise-resistant wavelet-based Bayesian fusion of multispectral and hyperspectral images," IEEE Trans. Geosci. and Remote Sens., vol. 47, no. 11, pp. $3834-3843$, Nov. 2009.

[11] Q. Wei, N. Dobigeon, and J.-Y. Tourneret, "Bayesian fusion of hyperspectral and multispectral images," in Proc. IEEE Int. Conf. Acoust., Speech, and Signal Processing (ICASSP), Florence, Italy, May 2014.

[12] _ _ "Bayesian fusion of multi-band images," arXiv preprint arXiv:1307.5996, 2013

[13] X. Otazu, M. Gonzalez-Audicana, O. Fors, and J. Nunez, "Introduction of sensor spectral response into image fusion methods. Application to wavelet-based methods," IEEE Trans. Geosci. and Remote Sens., vol. 43, no. 10, pp. 2376-2385, 2005.

[14] R. Molina, A. K. Katsaggelos, and J. Mateos, "Bayesian and regularization methods for hyperparameter estimation in image restoration," IEEE Trans. Image Process., vol. 8, no. 2, pp. 231-246, 1999.

[15] R. Molina, M. Vega, J. Mateos, and A. K. Katsaggelos, "Variational posterior distribution approximation in Bayesian super resolution reconstruction of multispectral images," Applied and Computational Harmonic Analysis, vol. 24, no. 2, pp. 251 $-267,2008$.

[16] A. P. Dawid, "Some matrix-variate distribution theory: notational considerations and a Bayesian application," Biometrika, vol. 68 , no. 1, pp. 265-274, 1981.

[17] A. Jalobeanu, L. Blanc-Feraud, and J. Zerubia, "An adaptive Gaussian model for satellite image deblurring," IEEE Trans. Image Process., vol. 13, no. 4, pp. 613-621, 2004.

[18] M. Xu, H. Chen, and P. K. Varshney, "An image fusion approach based on Markov random fields," IEEE Trans. Geosci. and Remote Sens., vol. 49, no. 12, pp. 5116-5127, 2011.

[19] J. M. Bioucas-Dias and J. M. Nascimento, "Hyperspectral subspace identification," IEEE Trans. Geosci. and Remote Sens., vol. 46, no. 8, pp. 2435-2445, 2008.

[20] P. J. Liu, "Using Gaussian process regression to denoise images and remove artefacts from microarray data," Ph.D. dissertation, University of Toronto, 2007.

[21] N. A. Woods, N. P. Galatsanos, and A. K. Katsaggelos, "Stochastic methods for joint registration, restoration, and interpolation of multiple undersampled images," IEEE Trans. Image Process., vol. 15, no. 1, pp. 201-213, Jan. 2006.

[22] C. P. Robert and G. Casella, Monte Carlo statistical methods. New York, NY, USA: Springer-Verlag, 2004.

[23] M. Bouriga and O. Féron, "Estimation of covariance matrices based on hierarchical inverse-Wishart priors," J. of Stat. Planning and Inference, 2012.

[24] C. P. Robert, The Bayesian Choice: from Decision-Theoretic Motivations to Computational Implementation, 2nd ed., ser. Springer Texts in Statistics. New York, NY, USA: SpringerVerlag, 2007.

[25] R. O. Green, M. L. Eastwood, C. M. Sarture, T. G. Chrien, M. Aronsson, B. J. Chippendale, J. A. Faust, B. E. Pavri, C. J. Chovit, M. Solis et al., "Imaging spectroscopy and the airborne visible/infrared imaging spectrometer (AVIRIS)," Remote Sens. of Environment, vol. 65, no. 3, pp. 227-248, 1998.

[26] D. Fleming, "Effect of relative spectral response on multispectral measurements and NDVI from different remote sensing systems," Ph.D. dissertation, University of Maryland, 2006.

[27] S. Li and B. Yang, "A new pan-sharpening method using a compressed sensing technique," IEEE Trans. Geosci. and Remote Sens., vol. 49, no. 2, pp. 738-746, Feb 2011.

[28] N. Yokoya, T. Yairi, and A. Iwasaki, "Coupled nonnegative matrix factorization unmixing for hyperspectral and multispectral data fusion," IEEE Trans. Geosci. and Remote Sens., vol. 50, no. 2, pp. 528-537, 2012.

[29] M. A. Bendoumi and M. He, "Unmixing approach for hyperspectral data resolution enhancement using high resolution multispectral image with unknown spectral response function," in IEEE Conference on Industrial Electronics and Applications (ICIEA). Melbourne, Australia: IEEE, 2013, pp. 511-515.

[30] Z. Chen, H. Pu, B. Wang, and G.-M. Jiang, "Fusion of hyperspectral and multispectral images: A novel framework based on generalization of pan-sharpening methods," IEEE Geosci. and Remote Sensing Lett., vol. 11, no. 8, pp. 1418-1422, Aug 2014 